\title{
Correlation between Clinical, Sonographic and Pathologic Findings of Patients Undergoing Appendectomy
}

\author{
Mahdokht Moghimi ${ }^{1}$, Borzoo Khaledifar', Abdolmajid Taheri' ${ }^{2}$, Forouzan Ganji ${ }^{3}$, Mahmoud Mobasheri ${ }^{4 *}$ \\ 1. Department of Surgery, Shahrekord University of Medical Sciences, Shahrekord, Iran \\ 2. Department of Radiology, Shahrekord University of Medical Sciences, Shahrekord, Iran \\ 3. Department of Social Medicine, Shahrekord University of Medical Sciences, Shahrekord, Iran \\ 4. Department of Epidemiology and Biostatistics, Faculty of Health, Shahrekord University of Medical Sciences, Shahrekord, Iran
}

*Corresponding Author: Mahmoud Mobasheri, Assistant Professor, Department of Epidemiology and Biostatistics, Faculty of health, Shahrekord University of Medical Sciences, Rahmatiyeh, Shahrekord, Iran

Email: mobasheri@skums.ac.ir

\begin{abstract}
Introduction: Acute appendicitis is the most common cause of acute abdomen and is considered as a surgical emergency. Approximately, $10 \%$ of patients with acute appendicitis and delayed diagnosis can face many complications. This study was designed to examine the correlation between ultrasound and pathological findings in patients with appendicitis and aimed to determine the diagnostic levels of ultrasound in these patients. Methods: This study is a retrospective study conducted on 500 patients with clinical symptoms suggestive of appendicitis in 2010-2012 in the Ayatollah Kashani hospital. These patients have undergone appendectomy. Having collected clinical and ultrasound data and compared them with pathological results, the data were analyzed.

Results: In this study, $56.3 \%$ of patients were male and $43.7 \%$ of them were female. The mean age of the study population was $24.8 \pm 14.1$ years. In this study, the sensitivity of ultrasonography in the diagnosis of acute appendicitis was $83 \%$ and its specificity was $56 \%$. The positive predictive value of ultrasound in this study was $89.2 \%$ and the negative predictive value was $42.6 \%$.

Conclusion: Based on these results and by comparing with those of other studies, the sensitivity and specificity of ultrasonography in the center is acceptable and is at the similar level to other centers. Besides, the accuracy of ultrasound in the negative cases is much more than the positive ones.

Keywords: Appendicitis, Sonography, Pathology, Sensitivity, Specificity
\end{abstract}

Article History: Received: 11 Mar 2015; Revised: 24 Apr 2015; Accepted: 30 Apr 2015

Cite this article as: Moghimi M, Khaledifar B, Taheri A, Ganji F, Mobasheri M. Correlation between clinical, sonographic and pathologic findings of patients undergoing appendectomy. Int J Travel Med Glob Health. 2015;3(2):59-63.

\section{Introduction}

Acute appendicitis is the most common cause of acute abdomen and appendectomy is the most common emergency surgery for appendicitis worldwide. About $10 \%$ of people get acute appendicitis which if not diagnosed on time will cause many complications in the patient. The main symptom of appendicitis is abdominal pain which, classically, is an umbilical pain that gradually moves to the Right Lower Quadrant (RLQ) of the abdomen $[1,2]$. Acute appendicitis is usually diagnosed according to the findings of the patient's history. The symptoms can occur in many forms and mimic the symptoms of other diseases; therefore, accurate and timely diagnosis of the disease requires special skills and experience [3]. As a result, the diagnosis of acute appendicitis in some cases can be a challenge and the use of imaging techniques is required. Advanced imaging techniques such as ultrasound and CT scan are used in the diagnosis of acute appendicitis. Use of these methods, especially in patients who have unspecified symptoms is very helpful [4]. Common forms of diagnostic and clinical utilization of other means to confirm the clinical diagnosis is popular in emergency centers [5]. Normal X-rays have little sensitivity for the diagnosis of appendicitis and typically is not recommended. Ultrasound is recommended in the evaluation of acute appendicitis. Ultrasound is the preferred method and more suitable to diagnose appendicitis and is widely used in medical centers [1]. This study attempts to assess the correlation between ultrasound and pathological findings in patients with appendicitis and to determine the diagnostic extent of ultrasound in these patients.

\section{Methods}

The present study is a retrospective study which is performed on 500 patients with clinical symptoms suggestive of appendicitis who underwent appendectomy in the Ayatollah Kashani hospital of Shahrekord in 2010-12. The sample size was of 500 patients who had the required criteria of the study. This sample size is selected based on the formula to estimate average results of similar studies, with $95 \%$ confidence and a maximum absolute error of 0.5 . The information obtained from the clinical examination (which results in suspicion to appendicitis) and also the results of the ultrasound and pathology as well as other demographic information obtained from the patient were recorded in the provided information form. The data gathering tool, reliability, validity and patient information forms were designed according to previous re- 
searches conducted in this area. Alvarado number is also calculated in this study according to valid references listed here. The inclusion criteria for this study were: undergoing appendectomy and ultrasound in the course of hospitalization before performing appendectomy, referring to a pathologist post-surgery. The exclusion criteria for this study were excessive obesity prior to the surgery that causes improper diagnosis, any problem that prevents the possibility of imaging including requiring emergency surgery, the lack of required information (at least, results for sonography and pathology) in the patients' clinical history.

Having collected data on clinical findings, ultrasound and compliance with pathological results, the data were analyzed. The results of this study have been analyzed using SPSS 16 software through the average standard deviation, relative abundance, Kappa coefficient and Chi-square test.

Findings from clinical ultrasound were corresponded with the results of pathological studies. Appendectomy patients were studied in terms of shift in pain, anorexia, nausea and vomiting, tenderness RLQ, rebound tenderness, fever, leukocytosis, and left shift.

Ultrasound in the supine position, the best position to assess the appendix, was performed using a linear probe with a frequency of 7.5-10 MHz and a gradual pressure technique.

A definite criterion for diagnosis of appendicitis was the presence of neutrophil cells within the muscular layer of the pathology specimens.

By using the data obtained from patients and the following formula, the Alvarado value was calculated and analyzed for patients (Table 1).

Table 1. Alvarado scoring system

\begin{tabular}{cccc}
\hline Alvarado Score & Demonstration & Value \\
\hline \multirow{2}{*}{ Signs } & Shift pain & 1 & 1 \\
& Anorexia & 1 & 2 \\
\multirow{2}{*}{ Symptoms } & Nausea and vomiting & 1 \\
& RLQ tenderness & 1 \\
Laboratory values & Rebound tenderness & 2 \\
\hline
\end{tabular}

Patients with the scores of 9 and 10 certainly have acute appendicitis. Patients with the scores of 7 and 8 are likely to have acute appendicitis. While those with the points of 5 and 6 do not contradict appendicitis, they are not diagnostic, either. In patients with the scores between 0 and 4 , the likelihood of appendicitis is low [1].

All the collected data about individuals were kept strictly confidential and were used only for research purposes. Ultrasound in the diagnosis and treatment processes was at the routine request of the physician; therefore, no cost was imposed to the patient. The results are reported collectively.

\section{Results}

In this study, $56.3 \%$ were male and $43.7 \%$ were female. The mean age of the study population is $14.1 \pm 24.8$ years. The Maximum and minimum age in this study was 89 and 3 years, respectively.

Clinical symptoms in patients who have undergone appendectomy, are as follows:

Laboratory data from the patients were collected to determine the presence of leukocytosis and left shift in the white blood cells. Table 2 and 3 shows the results.

Later in this study, using the formula given in Methods, the Lvardv value was calculated for all patients; the average of which is $2.52 \pm 6.42$ and it's highest and lowest are 9 and 0 , respectively.
Table 2. Frequency of clinical symptoms in appendectomized patients

\begin{tabular}{lcc}
\hline \multicolumn{1}{c}{ Title } & Number of Individuals & Percent \\
\hline RLQ tenderness & 435 & 89.5 \\
Rebound tenderness & 221 & 45.8 \\
Fever & 98 & 20.5 \\
Loss of appetite & 315 & 67.6 \\
Nausea and vomiting & 369 & 75.9 \\
Pain Shift & 230 & 48.4 \\
\hline
\end{tabular}

Table 3. Laboratory findings in appendectomized patients

\begin{tabular}{lcc}
\hline \multicolumn{1}{c}{ Title } & Number of Individuals & Percent \\
\hline Leukocytosis & 365 & 76.7 \\
Left shift & 347 & 74 \\
\hline
\end{tabular}

In order to facilitate the reviews, people were classified. According to this classification those who had an Lvardv number more than 5 were considered likely to have acute appendicitis and others were considered without this risk. Based on this classification, $77 \%$ of the patients were likely to develop appendicitis.

All the patients in this study underwent ultrasound examination to confirm the diagnosis pre-surgery. Based on the results, $75.7 \%$ of the people have had symptoms of acute appendicitis in ultrasonography; however, the results of $24.3 \%$ patients' ultrasound examination was negative.

The samples which were obtained after appendectomy surgery were sent to the pathology laboratory for a final confirmation and diagnosis. The results show that the pathology in $81.5 \%$ of the patients was positive and their acute appendicitis diagnosis was done properly. In the case of $18.5 \%$ of the people who have had surgery, their diagnosis of acute appendicitis were rejected. 
Ultrasound and pathology findings in patients who have undergone appendectomy are given in the table below (Table 4).

Table 4. Comparison of sonography's diagnosis per pathology results

\begin{tabular}{cccc}
\hline \multicolumn{2}{c}{ Pathology } & & \multicolumn{2}{c}{ Results } \\
Negative & Positive & Positive & Ultrasound \\
\hline $41(8.1 \%)$ & $340(67.6 \%)$ & Negative & \\
\hline $52(10.3 \%)$ & $70(13.9 \%)$ & Neger & \\
\hline
\end{tabular}

Based on statistical tests, sensitivity of ultrasound is $76.9 \%$ and its specificity is $27.8 \%$. False negatives are $17.8 \%$ and false positive are $16.6 \%$. Its positive predictive value is 78.1 $\%$ and reported negative predictive are $26.4 \%$.

Based on the Chi-square test, $\mathrm{P}$ value between sonographic findings and Lvardv value was 0.294.

\section{Discussion}

Acute appendicitis is considered to be the most common acute abdominal surgery. In many patients in need of surgery, clinical rating is a criterion for diagnosis and beginning treatments [6]. Usually, the diagnosis of acute appendicitis is performed based on the findings of the patients' history, clinical and laboratory data [3]. The accuracy of clinical diagnosis of acute appendicitis varies between $76 \%$ and $92 \%$. Due to the possibility of numerous complications as the result of delays in diagnosis, doctors have adopted a negative appendectomy rate of $15-25 \%[1,3]$. If we can reduce negative appendectomy cases with the help of imaging techniques such as ultrasonography, there would be a major advance in the management of these patients. Regarding the above-mentioned, in this study, clinical, ultrasound and pathology findings in patients who have undergone appendectomy were compared.

In this study, the mean age of the participants is $14.1 \pm 24.8$ years. In reference books and various studies done in this field, the second to fourth decades have been reported to be the most common age of appendicitis. The results of the present study also indicate these findigs.

In addition, in this study, similar to other studies, the percentage of men was higher compared to women.

In a study by Chandrasegaram et al., the acute appendicitis incidence was $71.3 \%$ and the positive appendectomy was $76.3 \%$, while negative appendectomy was significantly lower in men compared to women [7].

The main symptoms of acute appendicitis are per umbilical pain, anorexia, and nausea. Despite the technological advances in the preoperative diagnosis of acute appendicitis, the correct diagnosis can only be made in $76-92 \%$ of the patients $[8,9]$.

Leukocytosis is observed in $80-90 \%$ of appendicitis cases; however, the leukocyte number is below $18.000 \mathrm{~mm} 3$ unless perforation is present [10]. In a study by Hatipoglu et al. leukocyte counts were high in patients with acute and perforated appendicitis in $95 \%$ and $93 \%$, respectively [11].
The Alvarado score is utilized to determine the likelihood of appendicitis based on clinical signs, symptoms, and laboratory results. Among the 300 patients, $85.66 \%$ had Alvarado scores of 7 or less and $14.33 \%$ had scores greater than 7 . For patients with confirmed appendicitis, 25.7\% had Alvarado scores of 7 or less, whereas $93 \%$ had Alvarado scores greater than 7 [12].

In this study, the average value of Alvarado was $2.52 \pm 6.42$ and based on the Alvarado value, $77 \%$ of patients were likely to develop appendicitis. Looking at the results of other studies, we observed that the sensitivity and specificity of Alvarado were $74.8 \%$ and $68.4 \%$ respectively. Also, a negative appendectomy rate of 7 points or more, Alvarado criteria of $5.7 \%$, and a positive predictive value of $98.1 \%$ was observed. The diagnostic accuracy of the Alvarado criteria in points 7 and more was 74\% [13]. In Laal et al.'s study, diagnostic accuracy of the Alvarado score which was greater than six was reported as $88 \%$ and ultrasound results were $71.9 \%$ [14].

The diagnosis of appendicitis has been largely based on physical examination findings. During the last decade, the use of diagnostic imaging has superseded the clinical examination. The application of Ultrasound for the diagnosis of acute appendicitis in children was first described in 1981 and expanded during the next two decades [15-17]. In a pooled analysis, the ultrasound was reported as $85 \%$ sensitive and 94\% specific for diagnosing appendicitis [18]. Advantages include noninvasive nature and no risk of exposure to ionizing radiation $[16,19,20]$, decreased negative appendectomy rate, perforation rate, and lower costs $[15,21,22]$.

In this study, $75.7 \%$ of the cases had symptoms of acute appendicitis on ultrasound; however, the result of $24.3 \%$ of patients' ultrasound examination was negative. In a study conducted by Amanallahi et al., $12.7 \%$ of the patients had negative ultrasound results for the presence of appendicitis and $81.1 \%$ had a positive result for appendicitis [23]. In addition, in a study conducted by Mohammadi et al., ultrasound result of $67.8 \%$ was reported as acute appendicitis and $32.2 \%$ as normal [24]. Comparing the results obtained in this study with the similar studies, the performance of Ayatollah Kashani hospital radiology department was acceptable and parallel to other centers of the country.

Consistent with the results of histo-pathologic specimens, ultrasound results revealed that pathology of $81.5 \%$ of patients was positive for acute appendicitis, and that it was done properly. But for $18.5 \%$ of patients who had surgery, the diagnosis of acute appendicitis was rejected. In Amanallahi et al.'s study, $14.9 \%$ and $85.1 \%$ of the patients had negative and positive histological responses for appendicitis, respectively [23]. Sixty eight and 32percent of the patients undergoing appendectomy in Mohammadi et al.'s study were reported with acute appendicitis by histopathological findings, and normal appendicitis, respectively [24]. Considering the results of similar articles, misdiagnosis of acute appendicitis in Ayatollah Kashani's hospital were less than the other centers and had a more acceptable level. 
In this study, the sensitivity of ultrasonography in the diagnosis of acute appendicitis was $83 \%$ and its specificity was $56 \%$. The positive predictive value of ultrasound was $89.2 \%$ and the negative predictive value was $42.6 \%$. In a doubleblind prospective study, sensitivity, specificity, and accuracy of ultrasonography for acute appendicitis were $56.6 \%, 78.4 \%$, and $63 \%$, respectively. The positive and negative predictive values were $85.3 \%$ and $44.49 \%$, respectively [25].

In a meta-analysis, the pooled sensitivity and specificity were respectively $83 \%$ and $93 \%$ for the diagnosis of adult appendicitis [26]. False negativity rates of US are 10-32\% [27-31]. Falsely negative US results could lead to unnecessary delays in diagnosis and increased morbidity and cost. Lower pain scores and increased abdominal wall thickness are significantly associated with false negativity of US examination for acute appendicitis. False negativity is increasing among patients with higher BMI and shorter pain durations [32].

In a study by Amanallahi et al, sensitivity and specificity of ultrasonography were $3.98 \%$ and $4.76 \%$, respectively. Also, positive and negative predictive values of ultrasound were $95.7 \%$, and $89.4 \%$, respectively [23].

In the studies conducted in other parts of the world, similar results were obtained. According to the results obtained from a research conducted by Pacharn et al. in 2009 in Thailand, , negative predictive value of ultrasound was $95.1 \%$ [31].

In Chan et al.'s study, an ultrasonography accuracy of $92 \%$, sensitivity of $83 \%$ and specificity of $95 \%$ have been reported. Also in this study, positive and negative predictive values were calculated $86 \%$ and $94 \%$ respectively [33]. Also, in a study conducted by Gracey et al. in the United Kingdom in 2007 , the sensitivity of the diagnosis of appendicitis in general was $93.8 \%$ and the overall measured specificity was $91.3 \%$ [34].

The comparison of the sensitivity and specificity obtained in this study indicates that the sensitivity of ultrasonography in this center is similar to that of other centers both within and outside the country and is at an acceptable level. It can also be said that like other studies, the positive predictive value of sonographies was more than the negative predictive values. Therefore, at the time of positive ultrasound results, there is a very high chance of developing acute appendicitis.

Specificity and predictive values obtained in this study is slightly lower than that of other studies. The specificity and negative predictive values obtained in this study are reasonable and acceptable. Interpretation of the results of this study considering this point suggests that the sensitivity of ultrasonography in this center has been higher and mistakes were lower than in other centers.

Based on these results and comparing them with the results of other studies, the sensitivity and specificity of ultrasonography in this center is acceptable and has the same level as other centers. Also, the accuracy of ultrasound in positive cases has been much more than that of negative ones. As a result, after clinical suspicion of acute appendicitis in patients, false negative cases should be reduced by ultrasound to avoid unnecessary surgical procedures.

\section{Conclusions}

Considering ultrasound as an accurate diagnostic tool for assessing pathological processes in every part of the body is accepted worldwide. This is why it is used in most medical centers around the world as a routine diagnostic test. In general, the results of this study shows that in the diagnosis of appendicitis ultrasound is valuable and acceptable in the current situation of our society and we can use ultrasound to help diagnose patients with suspected acute appendicitis prior to surgery. Ultrasound can help with early detection and treatment which reduces the incidence of side effects and complications and on the other hand, negative appendectomy cases and hospital costs and avoids unnecessary surgery. Since ultrasound results depend on the skill of the operator, achieving such outcomes directly depends on the experience of the radiologist performing this technique and his familiarity with principles of the GI tract ultrasound.

\section{Suggestions}

1. Conducting long-term studies with larger sample sizes in order to monitor the accuracy, sensitivity and specificity of ultrasonography in the diagnosis of acute appendicitis

2. Conducting long-term studies with larger sample sizes in order to compare the effect of radiologist expertise in ultrasound

3. Conducting other researches by different radiologists and using their findings as diagnostic criteria in the case of being consistent with other findings

Limitations and problems of the implementation:

This study was very time-consuming and in some cases, recorded files were incomplete and necessary data were unavailable.

\section{Acknowledgements}

The present study is derived from a research project with grant no. 1123 which was approved by the Research Department of the Shahrekord University of Medical Sciences. The authors, hereby, express their appreciation to all financial and moral support provided by the department and the colleagues who have cooperated in this study.

\section{Authors' Contribution}

The authors were involved in the study design, data collection, interpretation of the results, and preparation of the manuscript.

\section{Funding/Support}

None received.

\section{Financial Disclosure}

The authors declared no financial disclosure. 


\section{References}

1. Jaffe MB, Berger HD. The Appendix. In: Brunicardi FC, editors. Schwart's Principle of Surgery. New York: Macgraz-Hill; 2010. p. 1073-93.

2. Goldin AB, Khanna P, Thapa M, McBroom JA, Garrison MM, Parisi MT. Revised ultrasound criteria for appendicitis in children improve diagnostic accuracy. Pediatr Radiol. 2011;41:993-99.

3. Maa J, Kirkwood KS. The Appendix. In: Courtney M, Townsend JR, editors. Sabiston the Text book Of Surgery the Biological Basis of modern surgical practice. Philadelphia: Saunders- Elsevier; 2010. p. 1333-48.

4. Bhatt M, Joseph L, Ducharme FM, Dougherty G, McGillivray D. Pro- spective validation of the Pediatric Appendicitis Score in a Canadian pe- diatric emergency department. Acad Emerg Med. 2009;16:591-6.

5. Widlus DM. CT scan for suspected acute appendicitis. J Community Hosp Intern Med Perspect. 2012;1(4):7.

6. Shin CS, Roh YN, Kim JI. Delayed appendectomy versus early appendectomy in the treatment of acute appendicitis: a retrospective study. World J Emerg Surg. 2014;9(1):8.

7. Chandrasegaram MD, Rothwell LA, An EI, Miller RJ. Pathologies of the appendix: a 10-year review of 4670 appendicectomy specimens. ANZ J Surg. 2012;82(11):844-7.

8. Andersson RE, Hugander A, Ravn H, Offenbartl K, Ghazi SH, Nyström PO, Olaison G. Repeated clinical and laboratory examinations in patients with an equivocal diagnosis of appendicitis. World J Surg. 2000;24:479-85.

9. Walker AR, Segal I. What causes appendicitis? J Clin Gastroenterol 1990;12:127-9.

10. Jaffe BM, Berger DH. Appendics. In: Brunicardi FC. Editors Schwartz's Principles of Surgery. 8th edition. New York: Mc Grawhill; 2004. p.1119-39.

11. Hatipoglu S, Hatipoglu F, Abdullayev R. Acute right lower abdominal pain in women of reproductive age: clinical clues. World J Gastroenterol. 2014;20(14):4043-9.

12. Kariman H, Shojaee M, Sabzghabaei A, Khatamian R, Derakhshanfar $\mathrm{H}$, Hatamabadi $\mathrm{H}$. Evaluation of the Alvarado score in acute abdominal pain. Ulus Travma Acil Cerrahi Derg. 2014;20(2):86-90.

13. Hooshmand B, Shokouh-Saremi A, Noferesti A, Yaghoubi MA, Elahifar A. Diagnostic accuracy of Alvarado score in the diagnosis of acute appendicitis. Zahedan J Res Med Sci. 2011;13(8):18-21.

14. Laal M, Granpaye L, Khodadi F, Salavatipour A, Sadeghi A R. Sonography versus the Alvarado Scoring System for the diagnosis of acute appendicitis. Tehran Univ Med J. 2008;66(6):408-12.

15. Sivit CJ. Imaging the child with right lower quadrant pain and suspected appendicitis: current concepts. Pediatr Radiol. 2004;34:44753

16. Rothrock SG, Pagane J. Acute appendicitis in children: emergency department diagnosis and management. Ann Emerg Med. 2000;36:39-51.

17. Smink DS, Finkelstein JA, Garcia Peña BM, Shannon MW, Taylor GA, Fishman SJ. Diagnosis of acute appendicitis in children using a clinical practice guideline. J Pediatr Surg. 2004;39:458-63.

18. Rasmussen OO, Hoffmann J. Assessment of the reliability of the symptoms and signs of acute appendicitis. J R Coll Surg Edinb.1991;36:372-7.

19. Hahn H, Hoepner FU, Kalle T, et al. Sonography of acute appendicitis in children: 7 years experience. Pediatr Radiol. 1998;28:147-51.

20. Schulte B, Beyer D, Kaiser C, Horsch S, Wiater A. Ultrasonography in suspected acute appendicitis in childhood-report of 1285 cases. Eur J Ultrasound. 1998;8:177-82.

21. Pen a BMG, Taylor GA, Fishman SJ, Mandl KD. Costs and effectiveness of ultrasonography and limited computed tomography for diagnosing appendicitis in children. Pediatrics. 2000;106:672-6.

22. Dilley A, Wesson D, Munden M, et al. The impact of ultrasound examinations on the management of children with suspected appendicitis: a 3-year analysis. J Pediatr Surg. 2001;36:303-8.

23. Amanallahi A, Rezaee M, Golpazir Sorkhe A. Comparing the results of abdominal ultrasonography and Alvarado clinical criteria in patients with suspected appendicitis and abdominal ultrasound effect of reducing the negative appendectomy. Kermanshah S J. 2012;16(1):723.

24. Mohammadi A, Khodabakhsh F. Sensitivity and Specificity of Ultrasonography in Diagnosis of Acute Appendicitis. Journal of Babo University of Medical Sciences. 2009;11(3):42-7.

25. Riazi A, Tavakoli Rishehri R, Karimi AA, Farzaneh MR, Eghbali SS Sonography in the diagnosis of acute Appendicitis. Iranian South Med J. 2003;6(1):37-44.
26. Doria AS, Moineddin R, Kellenberger CJ, Epelman M, Beyene J, Schuh S, Babyn PS, Dick PT. US or CT for diagnosis of appendicitis in children and adults? A meta-analysis. Radiology. 2006;241:83-94.

27. Balthazar EJ, Birnbaum BA, Yee J, Megibow AJ, Roshkow J, Gray C. Acute appendicitis: CT and US correlation in 100 patients. Radiology.1994;190:31-5

28. Gamanagatti S, Vashisht S, Kapoor A, Chumber S, Bal S. Comparison ofgraded compression ultrasonography and unenhanced spiral computed tomography in the diagnosis of acute appendicitis. Singapore Med J. 2007;48:80-7.

29. Jeffrey RB Jr, Laing FC, Townsend RR. Acute appendicitis: Sonographic criteria based on 250 cases. Radiology. 1988;167:327-9.

30. Mos C, Ile T, Halbac M, Chis L, Vesa S, Andor S. Ultrasonographic diagnosis of acute appendicitis. Med Ultrasonogr. 2009;11:7-18.

31. Pacharn P, Ying J, Linam LE, Brody AS, Babcock DS. Sonography in the evaluation of acute appendicitis: are negative sonographic findings good enough? J Ultrasound Med. 2010;29:1749-55.

32. Piyarom P, Kaewlai R. False-Negative appendicitis at ultrasound Nature and Association. Ultrasound Med Biol. 2014. Pii: S03015629(14)00087-8

33. Chan I, Bicknell SG, Graham M. Utility and diagnostic accuracy of sonography in detecting appendicitis in a community hospital. AJR Am J Roentgenol. 2005;184(6):1809-12.

34. Gracey D, McClure MJ. The impact of ultrasound in suspected acute appendicitis. Clin Radiol. 2007;62(6):573-8 . 